\title{
Equivalent Circuit of Piezoelectric Diaphragms for Impedance-Based Structural Health Monitoring Applications
}

\author{
Everaldo S. de Freitas, Member, IEEE, Fabricio G. Baptista, Member, IEEE, \\ Danilo E. Budoya, Member, IEEE, and Bruno A. de Castro, Member, IEEE
}

\begin{abstract}
Piezoelectric diaphragms are low-cost acoustic components commonly used in a wide variety of electronic devices, where the typical function is to generate an audible alarm. However, scientific study concerning the use of these components in advanced applications has increased in recent years because of their low cost and widespread availability in several standard sizes. Based on a growing interest in these components, we propose a basic equivalent electromechanical circuit considering a 1-D vibration assumption of piezoelectric diaphragms for structural health monitoring applications based on the electromechanical impedance (EMI) technique, which was used in this paper to assess the sensitivity of piezoelectric diaphragms to structural damage. The proposed circuit is a three-port type and is suitable for small metal structures. The sensitivities of three diaphragms of different sizes to structural damage were assessed using the proposed equivalent circuit and compared with the sensitivity of a conventional transducer commonly used in the EMI method. In addition, tests were performed on aluminum bars to experimentally validate the equivalent circuit, and the theoretical and experimental results correspond well.
\end{abstract}

Index Terms-Piezoelectric, diaphragm, equivalent circuit, impedance, SHM.

\section{INTRODUCTION}

$\mathbf{P}$ IEZOELECTRIC diaphragms are low-cost, compact and lightweight components manufactured by several companies and widely used in a variety of electronic devices. Piezoelectric diaphragms are the main element of acoustic components, informally known as "buzzers"; the main application of acoustic components is sound generation, such as audible alarms, telephone ringers, and piezoelectric speakers.

Manuscript received May 19, 2017; accepted July 4, 2017. Date of publication July 11, 2017; date of current version August 9, 2017. This work was supported by the São Paulo Research Foundation (FAPESP) under Grant 2015/02500-6, Grant 2015/24903-5, and Grant 2015/23272-1. The associate editor coordinating the review of this paper and approving it for publication was Dr. Stefan J. Rupitsch. (Corresponding author: Fabricio G. Baptista.)

E. S. de Freitas is with the São Paulo State University (UNESP), School of Engineering, Bauru, Department of Electrical Engineering, Laboratory of Transducers and Data Acquisition, Bauru 17033-360, Brazil, and also with the Department of Electrical Engineering and Automation, Unilins-University Center of Lins, Lins 16401-371, Brazil (e-mail: everaldo.freitas@feb.unesp.br).

F. G. Baptista, D. E. Budoya, and B. A. de Castro are with the São Paulo State University (UNESP), School of Engineering, Bauru, Department of Electrical Engineering, Laboratory of Transducers and Data Acquisition, Bauru 17033-360, Brazil (e-mail: fabriciogb@feb.unesp.br; danilo.budoya@feb.unesp.br; bruno.castro@feb.unesp.br).

Digital Object Identifier 10.1109/JSEN.2017.2725946
Although the main application has been sound generators, these components have also been used for more advanced applications because of their low cost and widespread availability in different sizes. One of the most common applications has been the use of these components as piezoelectric transducers for damage detection techniques in structural health monitoring (SHM) systems. This study theoretically analyzes piezoelectric diaphragms using a proposed equivalent electromechanical circuit for SHM applications with damage detection based on the electromechanical impedance (EMI) method.

The field of research in SHM [1] is scientifically and industrially relevant because there are many engineering structures in the world that require monitoring to detect incipient damage, such as cracks, corrosion, and loosened bolted connections, to guarantee the safety of people using the structures and reduce costs with preventive maintenance. Potential applications include bridges, pipelines, oil platforms, rotating machinery and large means of transport, such as aircraft and ships.

Damage must be detected by a minimally invasive technique to not alter the mechanical properties and operating conditions of the structure. Such techniques are commonly known as nondestructive evaluation (NDE) or nondestructive testing (NDT) [2]. There are many methods to detect damage or anomalies in the structure, such as those based on fiber optic sensors [3], acoustic emission [4], eddy currents [5], Lamb waves [6], [7], using electroluminescent strain sensor [8] and based on 3-D displacement measurements [9]. Among the various methods for detecting damage, the EMI technique [10], [11] is known for using thin and small piezoelectric ceramics operating simultaneously as actuators and sensors.

The main principle of the EMI method is based on the piezoelectric effect [12]. A piezoelectric transducer, such as a diaphragm attached to the host structure, simultaneously acts as a sensor (direct piezoelectric effect) and an actuator (reverse piezoelectric effect), establishing a relationship between the mechanical impedance of the structure and the electrical impedance of the transducer. By exciting the transducer at an appropriate frequency range and measuring the corresponding electrical impedance (or admittance), it is possible to detect variations in the mechanical impedance of the monitored structure caused by damage through changes in the electrical impedance, which is easy to measure. 
The piezoelectric transducers used in the EMI method have been lead zirconate titanate (PZT) ceramics [13], [14], which are typically very thin and can be cut into different sizes and shapes. Both sides of the PZT ceramics, also called piezoceramics, are coated with a thin metal film that acts as electrodes. Another commonly used transducer is macro fiber composite (MFC) [15], which has the advantage of being flexible and attachable to structures with uneven surfaces.

Recently, piezoelectric diaphragms have received attention in several scientific studies, such as loading monitoring [16], in a micro gas compressor [17], to detect partial discharges in power transforms [18], in an inertial measurement unit system [19], damage detection based on the EMI method [20], in grinding process monitoring [21] and for noninvasive detection of bio-signals [22]. A recent study [23] experimentally analyzed the feasibility of these components for SHM applications based on the EMI method. However, a theoretical analysis has not yet been reported in the literature.

Therefore, in this article, we propose a basic equivalent circuit based on a one-dimensional vibration assumption of a piezoelectric diaphragm to relate its electrical impedance to the mechanical properties of the monitored structure to assess the sensitivity to structural damage. The equivalent electromechanical circuit is presented in the next section.

\section{Equivalent Electromechanical Circuit}

Conventional PZT ceramics have been extensively studied for SHM applications, and several researchers have proposed one- [24], two- [25], and three- [26] dimensional analytical models and numerical models [27] based on the finite element method (FEM) to analyze the interactions between the PZT patch and monitored structure. Analytical models have also been proposed for piezoelectric diaphragms, such as models derived using the classical laminated plate theory [28], based on Hamilton's principle and the Rayleigh-Ritz method [29] and by dividing the diaphragm into two parts [30].

These proposed models obtain the exact vibration behavior of the diaphragm and determine resonance frequencies. In contrast, in the current study, we propose a basic electromechanical circuit based on a one-dimensional model specific to SHM applications based on the EMI method, which relates the electrical impedance of the piezoelectric diaphragm to the mechanical impedance of the monitored structure. The basic configuration of damage detection based on the EMI method is shown in Fig. 1 (a).

In Fig. 1 (a), a piezoelectric diaphragm, consisting of a circular piezoelectric element coated with a thin metal film (top electrode) mounted on a circular brass plate (bottom electrode), is attached to the monitored structure. There are several methods of installing piezoelectric transducers in the monitored structure [31]. In this study, the conventional installation method is considered, where the brass plate is fixed directly to the monitored structure using a highstiffness adhesive, such as cyanoacrylate or epoxy glue.

The monitored structure is considered a thin and narrow metal bar, which is the structure typically used in laboratories. In addition, the diaphragm is extremely thin, with a thickness on the order of a fraction of one millimeter. Under these

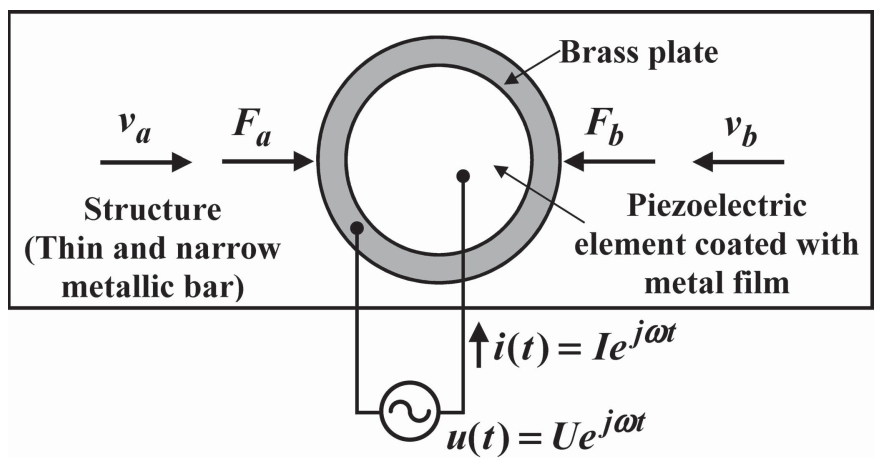

(a)

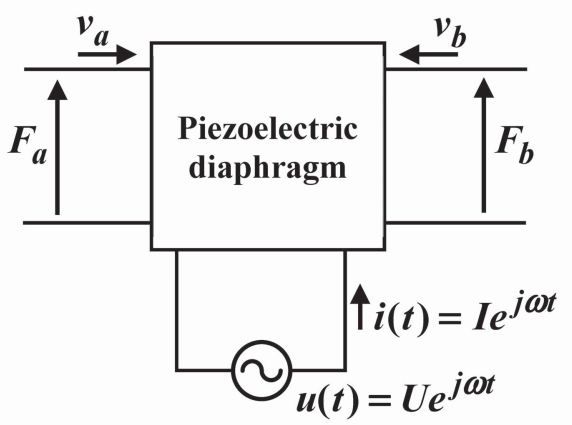

(b)

Fig. 1. (a) Piezoelectric diaphragm bonded to a thin, narrow metal bar and (b) its representation as a hexapole with one electrical port and two acoustic ports.

conditions, the principal vibration mode can be considered in the longitudinal direction of the structure for a one-dimensional assumption. Therefore, the piezoelectric diaphragm can be represented as a hexapole, as shown in Fig. 1 (b), with three ports: one electrical port and two acoustic ports, which is a solution commonly used in the literature [32]-[34].

As shown in Fig. 1 (a), exciting the diaphragm with an alternating voltage $u(t)$ with amplitude $U$ and angular frequency $\omega$ results in a current $i(t)$ with an amplitude $I$, two forces $F_{a}$ and $F_{b}$ in each acoustic port and two waves propagating at speeds $v_{a}$ and $v_{b}$ in the structure.

This one-dimensional assumption is appropriate only if certain conditions are satisfied: (a) the monitored structure is a simple narrow, thin bar and the transducer has a thickness on the order of a fraction of one millimeter, as discussed above; (b) the structure is metallic, and the damping effect can be disregarded; (c) the diaphragm is excited at a sufficiently low frequency, below 1 megahertz, such that the vibration toward its thickness can be disregarded [35]; (d) the amplitude of the excitation signal is low, and thus, the piezoelectric effect can be considered approximately linear [12]; and (e) the effects of the adhesive layer and metallic film (top electrode) can be disregarded because they are extremely thin. Therefore, the equivalent circuit proposed here does not account for the exact vibration behavior of the diaphragm and structure. The objective is to relate the electrical impedance of the diaphragm to the mechanical impedance of the structure and thus to assess the sensitivity to structural damage. An appropriate model 


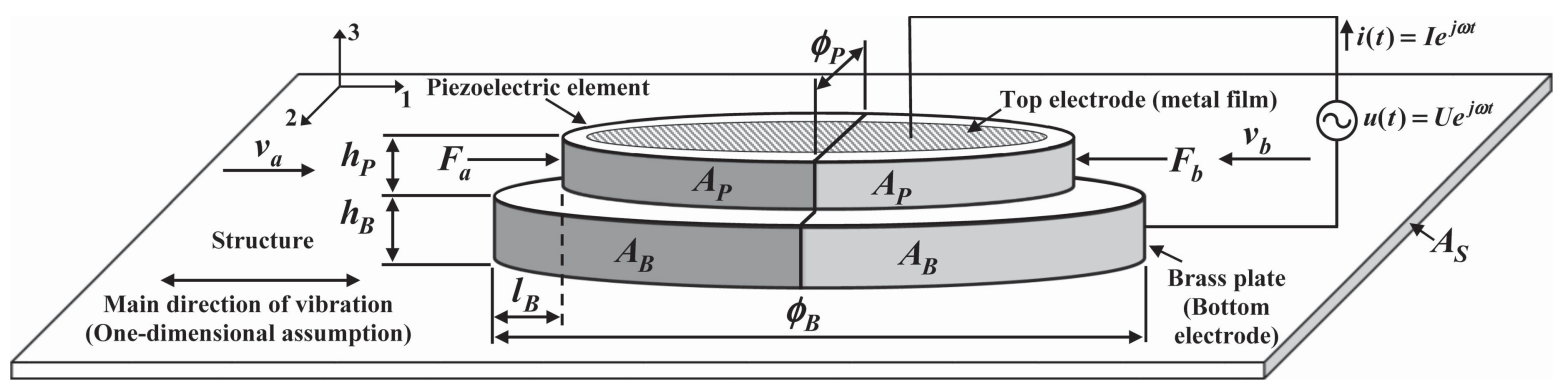

Fig. 2. Detailed representation of a piezoelectric diaphragm attached to the monitored structure.

based on more accurate solutions [28]-[30] must be derived for applications that do not meet the above conditions or when the exact vibration behavior is required.

Based on the above considerations, a more detailed representation of the piezoelectric diaphragm attached to the structure is shown in Fig. 2. As shown in Fig. 2, the transducer was divided into two structures, which is a solution similar to that proposed in [30], resulting in two acoustic ports, where the piezoelectric element consists of a PZT ceramic disc with diameter $\phi_{P}$ and thickness $h_{P}$ mounted on a circular brass plate with diameter $\phi_{B}$ and thickness $h_{B}$. Each part of the piezoelectric element and brass plate are semi circles with lateral area $A_{P}$ and $A_{B}$, respectively. The edge of the brass plate that exceeds the piezoelectric element has a length $l_{B}$. The transducer is attached to the monitored structure with a crosssectional area $A_{S}$. The constitutive piezoelectric equations are given by [12]

$$
\begin{aligned}
D_{i} & =d_{i k l} T_{k l}+\varepsilon_{i k}^{T} E_{k} \\
S_{i j} & =s_{i j k l}^{E} T_{k l}+d_{k i j} E_{k}
\end{aligned}
$$

where $E_{k}$ and $D_{i}$ are the electric field and electrical displacement components, respectively; $S_{i j}$ is the mechanical strain component; $T_{k l}$ is the mechanical stress component; $d_{i k l}$ and $d_{k i j}$ are the piezoelectric constants; $s_{i j k l}^{E}$ is the elastic compliance under a constant electric field; $\varepsilon_{i k}^{T}$ is the permittivity component at a constant stress; and the subscripts $i, j, k$, and $l$ represent the natural coordinate system of the piezoelectric crystal and take values of 1,2 , and 3 .

For a one-dimensional assumption in which the main direction of vibration is in the longitudinal direction of the structure and using simplified notation [12] for the indices, the basic constitutive equations for a PZT ceramic can be simplified as

$$
\begin{aligned}
D_{3} & =d_{31} T_{1}+\varepsilon_{33}^{T} E_{3} \\
S_{1} & =s_{11}^{E} T_{1}+d_{31} E_{3}
\end{aligned}
$$

Following a procedure similar to that presented in a previous study [34], the forces $F_{a}$ and $F_{b}$ on each acoustic port of the piezoelectric element shown in Fig. 2 can be approximated in terms of the amplitude $U$ of the excitation voltage as

$$
\begin{aligned}
F_{a} \cong & -j \frac{Z_{P}}{\sin \left(k_{P} \phi_{P}\right)}\left(v_{a}+v_{b}\right)+j Z_{P} \tan \left(\frac{k_{P} \phi_{P}}{2}\right) v_{a} \\
& +\frac{\pi \phi_{P} d_{31}}{2 s_{11}^{E}} U
\end{aligned}
$$

$$
\begin{aligned}
F_{b} \cong & -j \frac{Z_{P}}{\sin \left(k_{P} \phi_{P}\right)}\left(v_{a}+v_{b}\right)+j Z_{P} \tan \left(\frac{k_{P} \phi_{P}}{2}\right) v_{b} \\
& +\frac{\pi \phi_{P} d_{31}}{2 s_{11}^{E}} U
\end{aligned}
$$

where $v_{a}$ and $v_{b}$ are the speeds of the waves propagating in the structure, $\phi_{P}$ is the diameter of the piezoelectric element, $j$ is the unit imaginary number, $Z_{P}$ is the mechanical impedance of the piezoelectric element, and $k_{P}$ is the wavenumber.

The wave-number $k_{P}$ is given by

$$
k_{P}=\omega \sqrt{s_{11}^{E} \rho_{p}}
$$

where $\omega$ is the angular frequency of the excitation signal, and $\rho_{p}$ is the mass density of the PZT ceramic.

If the ceramic disc is small and the damping effect can be disregarded, then the mechanical impedance $Z_{P}$ is approximated as

$$
Z_{P} \cong A_{P} \sqrt{\frac{\rho_{p}}{s_{11}^{E}}}
$$

where $A_{P}=\pi \phi_{P} h_{P} / 2$ is the lateral area of the semicircle for each acoustic port, as shown in Fig. 2.

The amplitude $U$ of the excitation signal in terms of the electric current $I$ and waves with velocities $v_{a}$ and $v_{b}$ is approximated by

$$
U \cong \frac{1}{j \omega C_{0}}\left[I+\frac{\pi \phi_{P} d_{31}}{2 s_{11}^{E}}\left(v_{a}+v_{b}\right)\right]
$$

where $C_{0}$ is the static capacitance of the piezoelectric diaphragm, and the term $1 / j \omega C_{0}$ is the capacitive reactance.

Equations (5), (6) and (9) relate the mechanical quantities of the acoustic ports with the electrical quantities of the electrical port of the ceramic disc. Therefore, from these equations, we can represent the PZT ceramic disc by an electrical equivalent circuit, as shown in Fig. 3.

By applying Kirchhoff's laws in the circuit of Fig. 3 and relating to (5), (6) and (9), we obtain the following complex impedances

$$
\begin{aligned}
& Z_{P 1}=j Z_{P} \tan \left(\frac{k_{P} \phi_{P}}{2}\right) \\
& Z_{P 2}=-j \frac{Z_{P}}{\sin \left(k_{P} \phi_{P}\right)}
\end{aligned}
$$




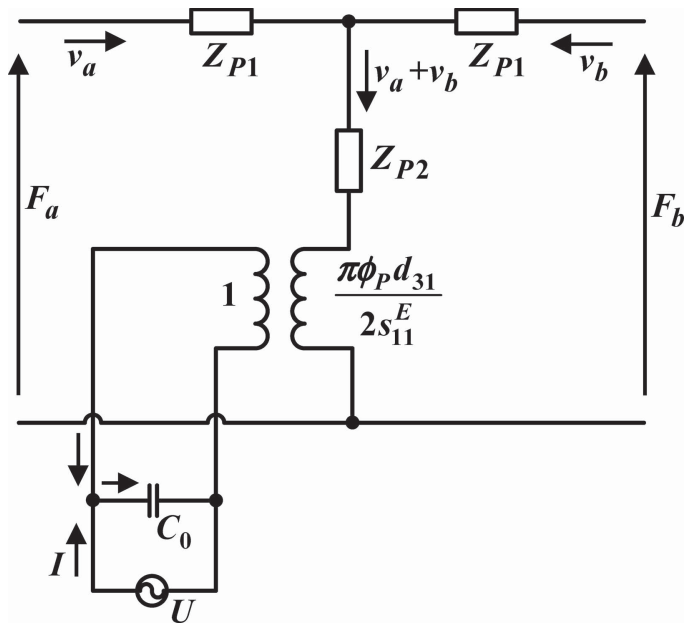

Fig. 3. Equivalent circuit of the piezoelectric element represented as a hexapole with two acoustic ports and an electric port.

According to the equivalent circuit, the piezoelectric element is essentially a capacitor with capacitance $C_{0}$. The electromechanical transformer with a transformation ratio equal to $1: \pi \phi_{P} d_{31} / 2 s_{11}^{E}$ performs the interaction with the mechanical quantities in the secondary. However, this circuit does not yet include the effects of the brass disc and monitored structure. The effect of brass paths between the ceramic and structure can be modeled with a similar circuit (except for the absence of the electromechanical transformer and electrical port) in cascade in each acoustic port, similarly as proposed in [36]. The structure is represented by its mechanical impedance $\left(Z_{S}\right)$. The complete equivalent circuit is shown in Fig. 4.

Similar to the impedances $Z_{P 1}$ and $Z_{P 2}$ for the piezoelectric element, the complex impedances $Z_{B 1}$ and $Z_{B 2}$ for the brass disc are given by

$$
\begin{aligned}
& Z_{B 1}=j Z_{B} \tan \left(\frac{k_{B} l_{B}}{2}\right) \\
& Z_{B 2}=-j \frac{Z_{B}}{\sin \left(k_{B} l_{B}\right)}
\end{aligned}
$$

where $l_{B}$ is the brass path between the PZT ceramic and monitored structure, as shown is Fig. 2, and $k_{B}$ is the wavenumber, given by

$$
k_{B}=\omega \sqrt{s_{B} \rho_{B}}
$$

where $s_{B}$ and $\rho_{B}$ are the elastic compliance and mass density of the brass disc, respectively.

For a small brass disc and disregarding the damping effect, the mechanical impedance $Z_{B}$ is approximately calculated as

$$
Z_{B} \cong A_{B} \sqrt{\frac{\rho_{B}}{s_{B}}}
$$

where $A_{B}=\pi \phi_{B} h_{B} / 2$ is the lateral area of the semicircle of the brass plate for each acoustic port, as shown in Fig. 2.

The electrical impedance of the piezoelectric diaphragm can be obtained by calculating the equivalent impedance from the terminals of the excitation voltage of the circuit in Fig. 4 as follows

$$
\begin{aligned}
& Z_{D}(\omega) \\
& =\frac{1}{j \omega C_{0}} \|\left\{\begin{array}{l}
\left(\frac{2 s_{11}^{E}}{\pi \phi_{P} d_{31}}\right)^{2} \\
\times\left[Z_{P 2}+\frac{1}{2}\left(Z_{P 1}+Z_{B 1}+\frac{Z_{B 2}\left(Z_{B 1}+Z_{S}\right)}{Z_{B 1}+Z_{B 2}+Z_{S}}\right)\right]
\end{array}\right\}
\end{aligned}
$$

where the symbol || indicates a parallel association.

According to (16), there is a relation between the electrical impedance $\left(Z_{D}(\omega)\right)$ of the piezoelectric diaphragm and the mechanical impedance $\left(Z_{S}\right)$ of the monitored structure. Therefore, variations in the mechanical impedance of the structure caused by damage, such as cracks, corrosion, and loosened bolted connections, can be detected by measuring the electrical impedance of the transducer.

For narrow and thin metal structures, the damping effect can be neglected, and the mechanical impedance $\left(Z_{S}\right)$ can be approximately given by

$$
Z_{S} \cong A_{S} \sqrt{\frac{\rho_{S}}{s_{S}}}
$$

where $s_{S}$ and $\rho_{S}$ are the elastic compliance and mass density of the monitored structure, respectively, and $A_{S}$ is the crosssectional area orthogonal to the longitudinal direction of the structure, as shown in Fig. 2.

The purpose of this study is to theoretically analyze the feasibility of piezoelectric diaphragms for damage detection based on the EMI method, comparing the results with those obtained from conventional PZT ceramics. For this purpose, we must obtain the electrical impedance of a PZT ceramic disc attached directly to the structure (without the brass disc). This can be achieved by disregarding the brass disc impedances in the circuit of Fig. 4, or equivalently, setting $Z_{B 1}=0$ and $Z_{B 2} \rightarrow \infty$ in (16). Thus, we obtain

$$
Z_{C}(\omega)=\frac{1}{j \omega C_{0}} \|\left[\left(\frac{2 s_{11}^{E}}{\pi \phi_{P} d_{31}}\right)^{2}\left(Z_{P 2}+\frac{Z_{P 1}+Z_{S}}{2}\right)\right]
$$

where $Z_{C}(\omega)$ is the electrical impedance of a conventional PZT ceramic disc with diameter $\phi_{P}$.

Equations (16) and (18) theoretically allow us to assess and compare the sensitivity of piezoelectric diaphragms and conventional PZT ceramics to structural damage detection. The theoretical sensitivity analysis is presented in the next section.

\section{Theoretical Analysis of the Sensitivity}

The main principle of damage detection based on the EMI method is to measure the electrical impedance of the piezoelectric transducer at an appropriate frequency range and to analyze the variations in the electrical impedance signatures caused by variations in the mechanical impedance of the monitored structure due to damage. Based on this principle and according to (16), the sensitivity of the piezoelectric diaphragm to structural damage can be assessed by analyzing the corresponding variation in its electrical 


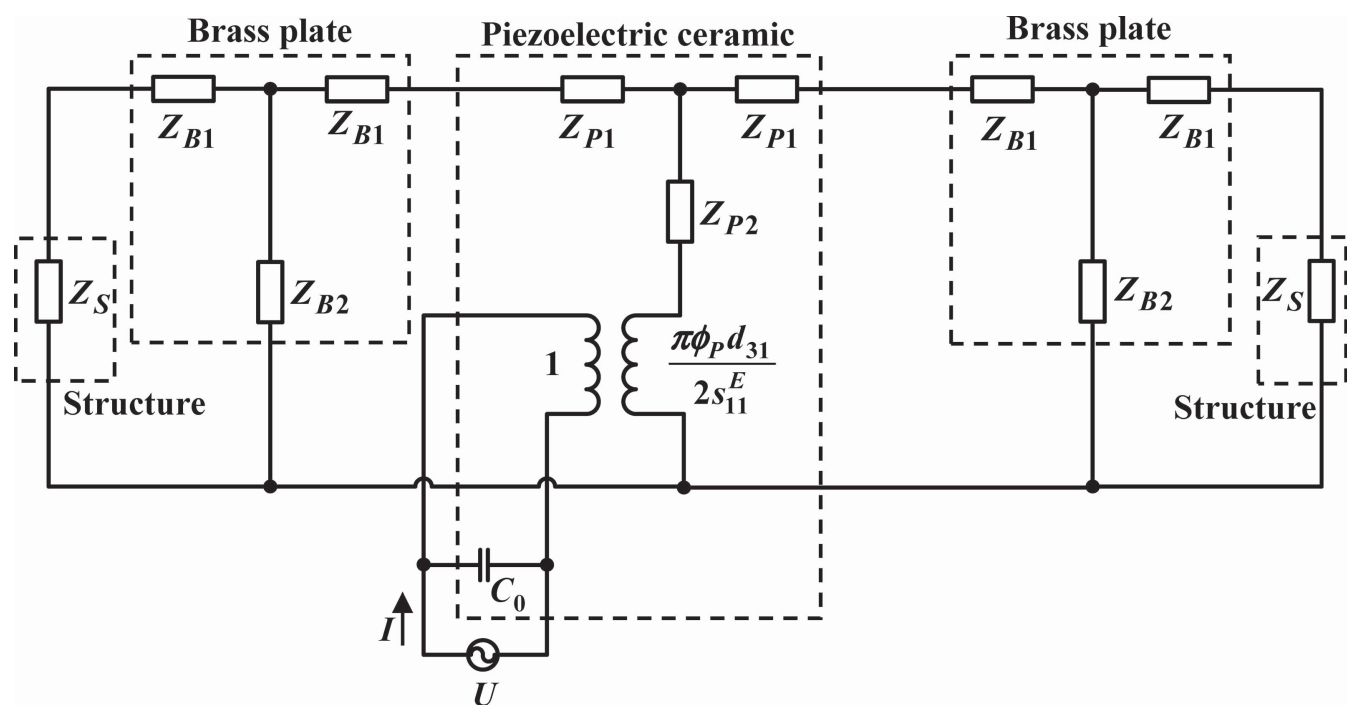

Fig. 4. Complete equivalent circuit of the piezoelectric diaphragm attached to the monitored structure.

TABLE I

Properties of the Piezoelectric DiaphragmS

\begin{tabular}{lccc}
\hline \hline Part number & $7 \mathrm{BB}-12-9$ & $7 \mathrm{BB}-20-6$ & $7 \mathrm{BB}-35-3$ \\
Brass plate diameter $\phi_{B}(\mathrm{~mm})$ & 12.0 & 20.0 & 35.0 \\
Brass plate thickness $h_{B}(\mathrm{~mm})$ & 0.10 & 0.20 & 0.30 \\
Brass mass density $\rho_{B}\left(10^{3} \mathrm{Kg} / \mathrm{m}^{3}\right)$ & 8.5 & 8.5 & 8.5 \\
Brass compliance $s_{B}\left(10^{-12} \mathrm{~m}^{2} / \mathrm{N}\right)$ & 9.1 & 9.1 & 9.1 \\
Ceramic disc diameter $\phi_{P}(\mathrm{~mm})$ & 9.0 & 14.0 & 25.0 \\
Ceramic disc thickness $h_{P}(\mathrm{~mm})$ & 0.12 & 0.22 & 0.23 \\
Ceramic mass density $\rho_{p}\left(10^{3} \mathrm{Kg} / \mathrm{m}^{3}\right)$ & 7.8 & 7.8 & 7.8 \\
Piezoelectric constant $d_{31}\left(10^{-12} \mathrm{~m} / \mathrm{V}\right)$ & -207 & -207 & -207 \\
Elastic compliance $s_{11}^{E}\left(10^{-12} \mathrm{~m}^{2} / \mathrm{N}\right)$ & 15.8 & 15.8 & 15.8 \\
Capacitance $C_{0}(\mathrm{nF})$ & 8.0 & 10.0 & 30.0 \\
\hline \hline
\end{tabular}

impedance $\left(Z_{D}(\omega)\right)$ due to the variation in the mechanical impedance of the monitored structure $\left(Z_{S}\right)$, which is a similar analysis as those reported in previous studies to select the optimal frequency bands to damage [34], for the proper transducer sizing [37] and to analyze the transducer loading effect [38].

Therefore, the sensitivity to damage can be theoretically assessed by the partial derivative of the function $Z_{D}(\omega)$ with respect to the variable $Z_{S}$ as follows

$$
\eta_{D}(\omega)=\left|\frac{\partial\left|Z_{D}(\omega)\right|}{\partial Z_{S}}\right|
$$

where $\eta_{D}(\omega)$ is the sensitivity of the piezoelectric diaphragm.

Similarly, the sensitivity to damage of a conventional PZT ceramic is given by

$$
\eta_{C}(\omega)=\left|\frac{\partial\left|Z_{C}(\omega)\right|}{\partial Z_{S}}\right|
$$

where $\eta_{C}(\omega)$ is the sensitivity of the conventional PZT ceramic, and $Z_{C}(\omega)$ is its electrical impedance, given by (18).
TABLE II

Properties of the Conventional Ceramic T107-A4E-273

\begin{tabular}{lc}
\hline \hline Diameter $\phi_{P}(\mathrm{~mm})$ & 12.7 \\
Thickness $h_{P}(\mathrm{~mm})$ & 0.191 \\
Piezoelectric constant $d_{31}\left(10^{-12} \mathrm{~m} / \mathrm{V}\right)$ & -190 \\
Elastic compliance $s_{11}^{E}\left(10^{-12} \mathrm{~m}^{2} / \mathrm{N}\right)$ & 15.2 \\
Capacitance $C_{0}(\mathrm{nF})$ & 10.6 \\
Mass density $\rho_{p}\left(10^{3} \mathrm{Kg} / \mathrm{m}^{3}\right)$ & 7.8 \\
\hline \hline
\end{tabular}

Three piezoelectric diaphragm models were considered in this study, 7BB-12-9, 7BB-20-6 and 7BB-35-3, manufactured by Murata Electronics North America, Inc. (Smyrna, GA, USA), with brass plate diameters of 12,20 and $35 \mathrm{~mm}$, respectively. According to the results, diaphragms with up to $35 \mathrm{~mm}$ in diameter are small enough for a one-dimensional assumption and disregard the damping effect. A conventional circular PZT ceramic model T107-A4E-273 manufactured by Piezo Systems, Inc. (Woburn, MA, USA) was used as a reference, and the results were compared with the results obtained for the piezoelectric diaphragms. The dimensions and properties of the diaphragms [39] and the conventional ceramic [40] are shown in Tables I and II, respectively.

By substituting the dimensions and properties of the two types of transducers in the equations of their electrical impedances given by (16) and (18), the sensitivities to structural damage can be assessed and compared using (19) and (20). Fig. 5 shows the relative sensitivities obtained following this procedure.

In Fig. 5, the sensitivities are plotted as a function of the frequency and the mechanical impedance of the monitored structure, which is normalized with respect to the mechanical impedance of the transducer $\left(Z_{S} / Z_{p}\right.$ ratio). In addition, the sensitivities are normalized assuming that the highest value 

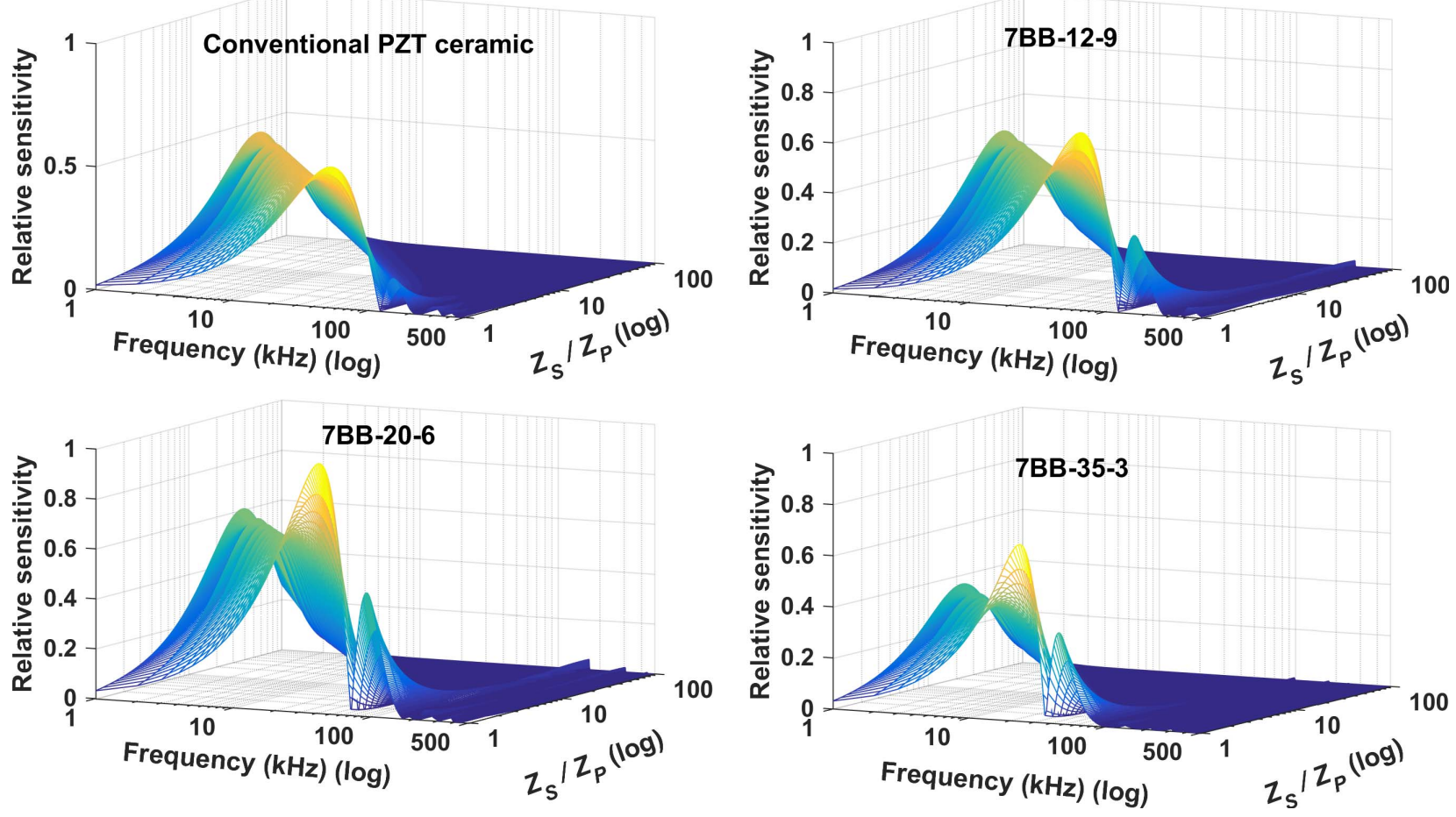

Fig. 5. Comparison of the relative sensitivities of the conventional PZT ceramic and piezoelectric diaphragms.

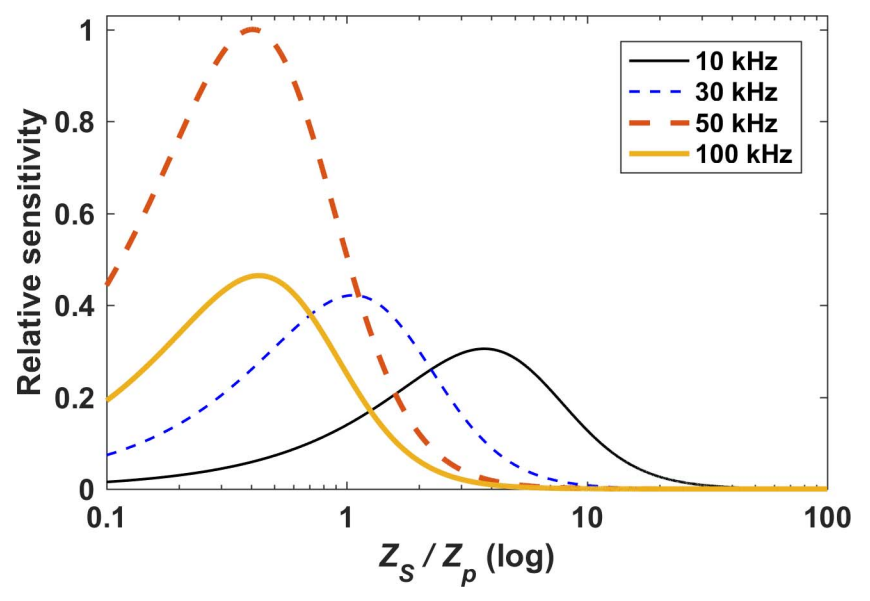

Fig. 6. Sensitivity to damage as a function of the impedance ratio for different fixed frequencies.

is 1 , which was obtained with the 20 -mm-diameter diaphragm (7BB-20-6).

The conventional ceramic and the three diaphragm models exhibit similar behaviors. The sensitivity to damage is higher at low frequencies and tends to decrease with increasing frequency, particularly when the mechanical impedance of the structure is large compared to the mechanical impedance of the transducer, that is, a high $Z_{S} / Z_{p}$ impedance ratio.

The effect of the impedance ratio on the sensitivity to damage is better illustrated in Fig. 6, which presents the sensitivities as a function of the $Z_{S} / Z_{p}$ ratio at different fixed frequencies obtained for the 7BB-20-6 diaphragm. The results obtained are similar for the other diaphragm models and the conventional ceramic. As shown in Fig. 6, for each frequency at which the transducer is excited, there is an optimal value for the impedance ratio that provides a high sensitivity. Regardless of the frequency, the sensitivity tends to decrease for small or large impedance ratios, as is also observed in the sensitivities shown in Fig. 5.

Another parameter that affects the sensitivity to damage is the equivalent electromechanical transformer, as indicated in the circuits shown in Fig. 3 and 4. A transformation ratio close to 1 allows for an effective bidirectional relationship between the electrical quantities of the transducer and the mechanical quantities of the monitored structure, that is, a better relationship between the electrical impedance of the transducer and the mechanical impedance of the structure, which is desirable for damage detection. The transformation factor in the secondary $\left(\pi \phi_{P} d_{31} / 2 s_{11}^{E}[\mathrm{~N} / \mathrm{V}]\right)$ is directly proportional to the diameter of the piezoelectric element $\left(\phi_{P}\right)$ of the diaphragm. Therefore, the transformation ratio increases with increases in the piezoelectric element diameter, as shown in Fig. 7.

According to Fig. 7, the transformation factor increases from approximately $0.2 \mathrm{~N} / \mathrm{V}$ to approximately $0.8 \mathrm{~N} / \mathrm{V}$ as the diameter of the piezoelectric element increases from $10 \mathrm{~mm}$ to $40 \mathrm{~mm}$, which is desirable. In contrast, increasing the diameter of the piezoelectric element also increases the capacitance of the transducer and thus decreases its reactance, which is undesirable. The variation of the reactive capacitance relative to the diameter at a frequency of $50 \mathrm{kHz}$ is shown in Fig. 7 as an example. For the same diameter variation of the previous case, the capacitive reactance decreases from approximately $480 \Omega$ to approximately $30 \Omega$; low capacitive reactance affects the sensitivity of the transducer to damage. As illustrated by the circuit in Fig. 4 and in (16), the electrical 


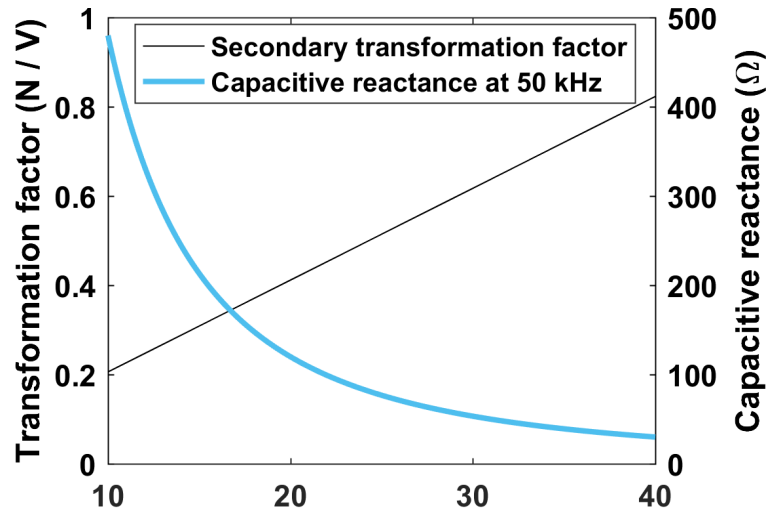

Diameter of the piezoelectric element $(\mathrm{mm})$

Fig. 7. Transformation factor and capacitive reactance (at $50 \mathrm{kHz})$ as a function of the diameter of the piezoelectric element.

impedance of the transducer consists of a capacitive reactance in parallel with the electromechanical transformer. A low capacitive reactance reduces the variation in the electrical impedance of the transducer $\left(Z_{D}(\omega)\right)$ due to the variation in the mechanical impedance of the structure $\left(Z_{S}\right)$ caused by damage.

We performed a basic experimental validation using aluminum bars to analyze the effectiveness of the proposed equivalent circuit. The experimental setup is presented in the next section.

\section{EXPERIMENTAL SETUP}

Experimental tests were performed on aluminum bars with dimensions of $500 \times 38.10 \times 3.18 \mathrm{~mm}$. These bars are thin and narrow and are thus appropriate for evaluating the proposed equivalent circuit based on a one-dimensional model and assessing the sensitivity of the piezoelectric diaphragms to structural damage.

The conventional PZT ceramic model T107-A4E-273 and the three piezoelectric diaphragms models, 7BB-12-9, 7BB-20-6 and 7BB-35-3, as presented in the previous section, were fixed at a distance of $20 \mathrm{~mm}$ (considering the center of each transducer) from the end of each bar using cyanoacrylate glue. The transducers attached to the aluminum bars are shown in Fig. 8.

Structural damage was induced in the bars by adding a metal mass (steel nut of $4 \times 2 \mathrm{~mm}$ and $1 \mathrm{~g}$ ) at a distance of $100 \mathrm{~mm}$ from the transducer. The addition of mass alters the mechanical impedance of the structure and can be related to damage. This procedure has the advantage of not causing permanent damage to the specimens.

The electrical impedance signatures of the transducers were measured via the alternative measurement system proposed in [41] using a NI-USB-6361 data acquisition (DAQ) device and a personal computer (PC). As reported in [41], the alternative system has good accuracy and precision, and provides impedance signatures rapidly with resonance peaks and amplitudes similar to a conventional impedance analyzer. The system was configured to excite the transducers with a chirp signal having an amplitude of $1 \mathrm{~V}$; the impedance signatures were obtained in a frequency range from 0 to $500 \mathrm{kHz}$ with a frequency step of $2 \mathrm{~Hz}$. All measurements were taken at a temperature of approximately $25^{\circ} \mathrm{C}$ controlled by an air conditioner because the impedance of the piezoelectric transducers is significantly temperature dependent [42] and with the aluminum bars resting on a bench on rubber blocks to avoid effects of external vibrations.

Damage was identified and quantified using the root-meansquare deviation (RMSD) damage index, which compares two electrical impedance signatures, where one of the signatures is obtained when the structure is considered in a healthy condition, commonly known as the baseline. The RMSD index is given by [1]

$$
R M S D=\sum_{\omega=\omega_{I}}^{\omega_{F}} \sqrt{\frac{\left[\operatorname{Re}\left(Z_{2}(\omega)\right)-\operatorname{Re}\left(Z_{1}(\omega)\right)\right]^{2}}{\operatorname{Re}\left(Z_{1}(\omega)\right)^{2}}}
$$

where $\operatorname{Re}\left(Z_{1}(\omega)\right)$ is the real part of the baseline signature, $\operatorname{Re}\left(Z_{2}(\omega)\right)$ is the real part of the signature after the damage, and RMSD is the index calculated in the frequency range with an initial frequency $\omega_{I}$ and final frequency $\omega_{F}$. The real part of the impedance signatures is typically more reactive to damage or changes in the structure's integrity [1].

In this study, we used the RMSD index to experimentally assess the sensitivity of the piezoelectric diaphragms and the conventional PZT ceramic and compare the results with the theoretical sensitivities obtained using the equivalent circuit. The experimental results are presented in the next section.

\section{EXPERIMENTAL RESULTS}

Before analyzing the experimental results, the theoretical sensitivities of the transducers can be assessed for the aluminum bar used in the experiments. Considering the dimensions of the bar presented in the previous section, its approximate mechanical impedance $\left(Z_{S}\right)$ can be calculated using (17) and the properties of the aluminum, which are mass density $\rho_{S}=2.7 \times 10^{3} \mathrm{~kg} / \mathrm{m}^{3}$ and elastic compliance $s_{S}=14.5 \times 10^{-12} \mathrm{~m}^{2} / \mathrm{N}$. With the mechanical impedance of the structure known, the sensitivity to damage of each transducer can be obtained from the general sensitivities shown in Fig. 5 considering the specific impedance ratios $\left(Z_{S} / Z_{p}\right)$ for each transducer. The relative sensitivities of the transducers for the aluminum bar are shown in Fig. 9.

As shown in Fig. 9, the sensitivity of the transducers to damage is high at low frequencies and tends to decrease significantly at high frequencies. In general, the 7BB-20-6 diaphragm (external diameter of $20 \mathrm{~mm}$ ) exhibits the highest sensitivity, followed by the conventional PZT ceramic, which has the second highest sensitivity and is similar in size to this diaphragm. The 7BB-12-9 diaphragm, which is the smallest transducer analyzed (external diameter of $12 \mathrm{~mm}$ ), has the lowest sensitivity throughout nearly the entire frequency range.

Although the 7BB-35-3 diaphragm is the largest transducer analyzed (external diameter of $35 \mathrm{~mm}$ ), its sensitivity to damage is lower than the sensitivity of the 7BB-20-6 diaphragm and the conventional ceramic. This result is consistent with the analysis shown in Fig. 7, which compares the transformation factor of the secondary of the electromechanical transformer 


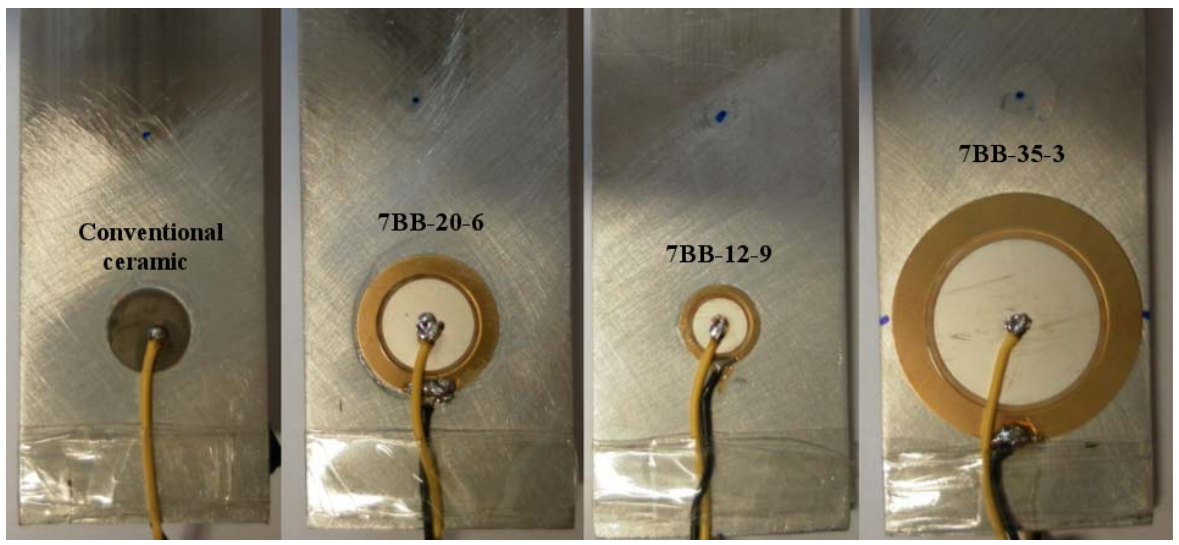

Fig. 8. Conventional PZT ceramic and piezoelectric diaphragms attached to aluminum bars.

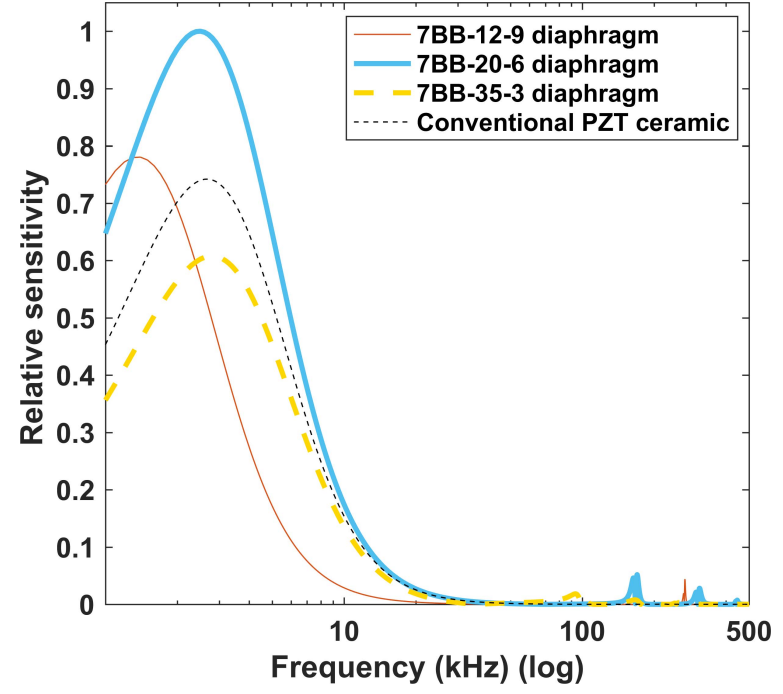

Fig. 9. Relative sensitivity of the transducers for the specific aluminum bar used in the experiment.

of the equivalent circuit with the capacitive reactance of the transducer with respect to the diameter of the piezoelectric element. Although the 7BB-35-3 diaphragm has a high transformation factor and provides a low mechanical impedance ratio $\left(Z_{S} / Z_{p}\right)$, its capacitive reactance is low compared to those of the other transducers. For frequencies above $40 \mathrm{kHz}$, the relative sensitivity is significantly low for all transducers, except for the 7BB-20-6 and 7BB-12-9 diaphragms, which have higher sensitivity for certain frequency bands.

The experimental electrical impedance signatures of the transducers are related to their theoretical sensitivities to damage. As an example, Fig. 10 (a) shows a comparison between the real part of the impedances of the transducers within the frequency range of $20-25 \mathrm{kHz}$, and Fig. 10 (b) shows the same signatures within the frequency range of $225-230 \mathrm{kHz}$.

For the signatures at the low frequencies shown in Fig. 10 (a), the conventional ceramic and the 7BB-20-6 diaphragm exhibit similar impedance signatures with more pronounced resonance peaks compared to the signatures of the other diaphragms. As is well known in the EMI method,

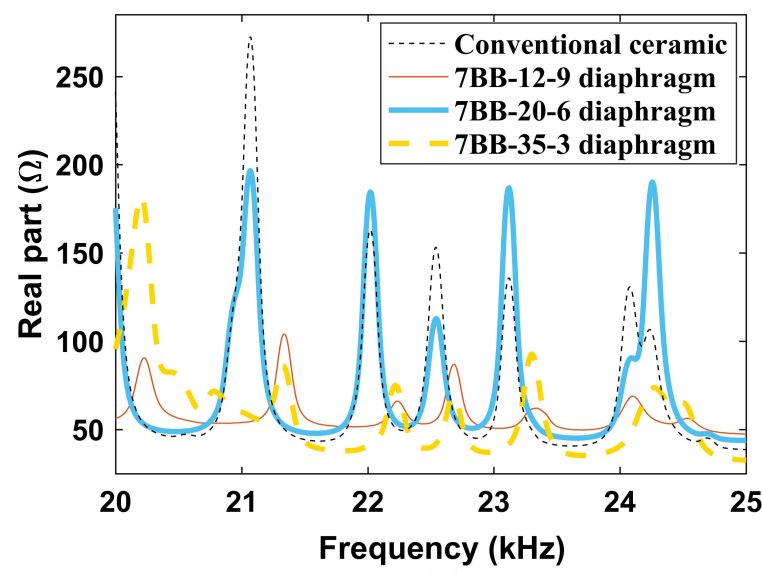

(a)

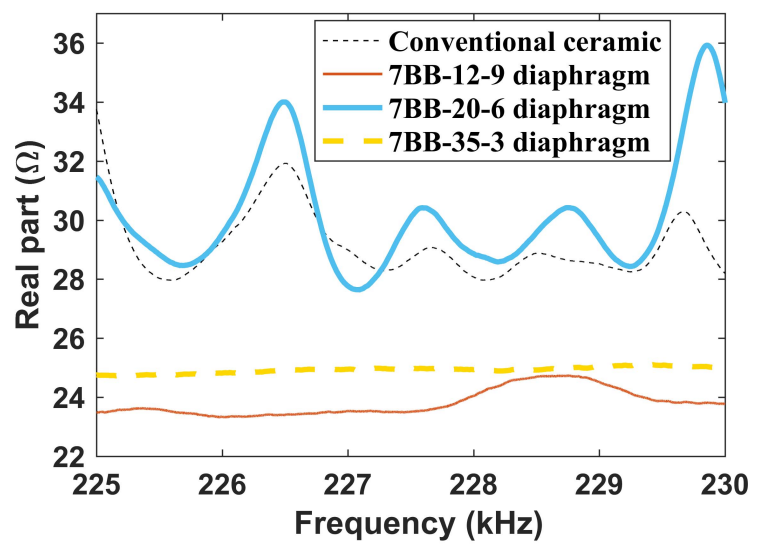

(b)

Fig. 10. Real part of the electrical impedance signatures at (a) low frequencies and (b) high frequencies.

more pronounced resonance peaks typically indicate higher sensitivity to damage. Therefore, these results are consistent with the relative theoretical sensitivities shown in Fig. 9. For high frequencies, the 7BB-20-6 diaphragm generally has an impedance signature with more pronounced resonance peaks, and the 7BB-12-9 and 7BB-35-3 diaphragms have signatures with extremely small peaks, as shown in Fig. 10 (b). These results are also consistent with the theoretical sensitivities.

A more formal procedure to experimentally assess the sensitivity of the transducers is by calculating the 


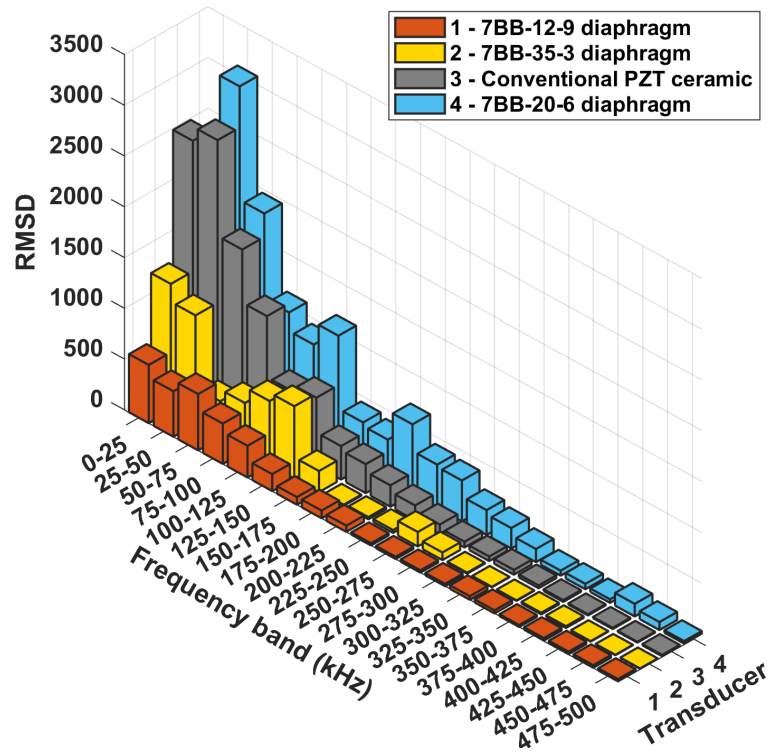

Fig. 11. Comparison of the RMSD indices obtained for the transducers.

RMSD index, which indicates the variation in the impedance signatures due to structural damage. This index was calculated using (21); damage was induced in the aluminum bars by the addition of mass according to the procedure described in the previous section. The RMSD index was calculated on subbands of $25 \mathrm{kHz}$ to allow for a comparison with the theoretical sensitivities over the entire frequency range. The results are shown in Fig. 11.

Similar to the theoretical sensitivities, the RMSD indices obtained for the transducers shown in Fig. 11 exhibit high values at low frequency bands and tend to decrease significantly at high frequency bands, although this decrease is not as abrupt as that observed in the theoretical sensitivities. The 7BB-20-6 diaphragm has the highest sensitivity to damage, and the 7BB-12-9 diaphragm has the lowest sensitivity to damage. The conventional PZT ceramic has the second highest sensitivity to damage, followed by the 7BB-35-3 diaphragm. These results are consistent with the theoretical sensitivities shown in Fig. 9. However, the high theoretical sensitivities of the 7BB-20-6 and 7BB-12-9 diaphragms in certain highfrequency sub-bands were not experimentally observed in the RMSD indices.

Despite some discrepancies, the experimental results correlate well with the theoretical results, indicating that the proposed equivalent circuit and sensitivity assessment are appropriate for the experimental conditions considered in this study.

\section{CONClusions}

This study theoretically analyzed the feasibility of piezoelectric diaphragms for impedance-based damage detection using a proposed equivalent electromechanical circuit. The proposed circuit was validated with basic experimental tests on aluminum bars; the experimental results were consistent with the theoretical results despite some discrepancies. Although the piezoelectric diaphragms have an additional brass disc in their construction, the results reported in this study conclusively indicate that their sensitivity to damage is not affected when compared to conventional PZT ceramics of similar dimensions, at least under the experimental conditions and one-dimensional assumptions considered in this study.

Although the proposed circuit is based on a one-dimensional vibration assumption and suitable only for narrow metal structures, the results reported in this article are important because they allow for an analytical comparison between diaphragms and conventional ceramics, in addition to analyzing the effect of the transducer diameter on the sensitivity to damage. An appropriate electromechanical model for effective applications in large structures must be developed in future research.

\section{ACKNOWLEDGMENT}

The authors are grateful to the anonymous reviewers for their valuable recommendations for improving this article.

\section{REFERENCES}

[1] C. R. Farrar and K. Worden, Structural Health Monitoring: A Machine Learning Perspective. Chichester, U.K.: Wiley, 2013.

[2] V. Giurgiutiu, "Embedded NDT with piezoelectric wafer active sensors," in Nondestructive Testing of Materials and Structures, vol. 6, O. Güneş and Y. Akkaya, Dordrecht, The Netherlands: Springer, 2013, pp. 987-992.

[3] M. Petrovic et al., "Intensity fiber-optic sensor for structural health monitoring calibrated by impact tester," IEEE Sensors J., vol. 16, no. 9 , pp. 3047-3053, May 2016.

[4] H. Yoo, Z. Liu, N. N. Quang, S. Kim, and F. Bien, "Implementation of nondestructive crack detection system for automotive Press panel," IEEE Sensors J., vol. 16, no. 2, pp. 383-389, Jan. 2016.

[5] X. Li, B. Gao, W. L. Woo, G. Y. Tian, X. Qiu, and L. Gu, "Quantitative surface crack evaluation based on eddy current pulsed thermography," IEEE Sensors J., vol. 17, no. 2, pp. 412-421, Jan. 2017.

[6] M. S. Salmanpour, Z. S. Khodaei, and M. H. Aliabadi, "Instantaneous baseline damage localization using sensor mapping," IEEE Sensors J., vol. 17, no. 2, pp. 295-301, Jan. 2017.

[7] Z. Shen, S. Chen, L. Zhang, K. Yao, and C. Y. Tan, "Direct-write piezoelectric ultrasonic transducers for non-destructive testing of metal plates," IEEE Sensors J., vol. 17, no. 11, pp. 3354-3361, Jun. 2017.

[8] J. Xu and H. Jo, "Development of high-sensitivity and low-cost electroluminescent strain sensor for structural health monitoring," IEEE Sensors J., vol. 16, no. 7, pp. 1962-1968, Apr. 2016.

[9] O. Kypris and A. Markham, "3-D displacement measurement for structural health monitoring using low-frequency magnetic fields," IEEE Sensors J., vol. 17, no. 4, pp. 1165-1174, Feb. 2017.

[10] T. Kamas, V. Giurgiutiu, and B. Lin, "E/M impedance modeling and experimentation for the piezoelectric wafer active sensor," Smart Mater. Struct., vol. 24, no. 11, p. 115040, Oct. 2015.

[11] M. I. Albakri and P. A. Tarazaga, "Electromechanical impedance-based damage characterization using spectral element method," J. Intell. Mater. Syst. Struct., vol. 28, no. 1, pp. 63-77, 2017.

[12] IEEE Standard on Piezoelectricity, IEEE Standard 176, 1988.

[13] V. G. M. Annamdas and M. A. Radhika, "Electromechanical impedance of piezoelectric transducers for monitoring metallic and non-metallic structures: A review of wired, wireless and energy-harvesting methods," J. Intell. Mater. Syst. Struct., vol. 24, no. 9, pp. 1021-1042, 2013.

[14] V. Giurgiutiu, "Piezoelectric wafer active sensors for structural health monitoring: State of the art and future directions," in Proc. Conf. Pressure Vessels Piping Division, Bellevue, WA, USA, Jul. 2010, pp. 1115-1121.

[15] A. J. Brunner, M. Birchmeier, M. M. Melnykowycz, and M. Barbezat, "Piezoelectric fiber composites as sensor elements for structural health monitoring and adaptive material systems," J. Intell. Mater. Syst. Struct., vol. 20, no. 9, pp. 1045-1055, 2009. 
[16] V. G. M. Annamdas and C. K. Soh, "Load monitoring using a calibrated piezo diaphragm based impedance strain sensor and wireless sensor network in real time," Smart Mater. Struct., vol. 26, no. 4, p. 045036 , Mar. 2017.

[17] S. Le and H. Hegab, "Investigation of a multistage micro gas compressor cascaded in series for increase pressure rise," Sens. Actuators A, Phys., vol. 256, pp. 66-76, Apr. 2017.

[18] B. Castro et al., "Partial discharge monitoring in power transformers using low-cost piezoelectric sensors," Sensors, vol. 16, no. 8, p. 1266, 2016.

[19] H. Almabrouk, M. Kharroubi, F. Tounsi, B. Mezghani, and Y. Bernard, "Macro model analysis of a single mass 6-DOF inertial measurement unit system," in Proc. 11th Int. Design Test Symp. (IDT), Hammamet, Tunisia, Dec. 2016, pp. 290-295.

[20] H. A. Tinoco and D. J. Marulanda, "Damage identification in active plates with indices based on Gaussian confidence ellipses obtained of the electromechanical admittance," J. Nondestr. Eval., vol. 34, no. 3 , p. 28 , Sep. 2015.

[21] M. Marchi, F. G. Baptista, P. R. de Aguiar, and E. C. Bianchi, "Grinding process monitoring based on electromechanical impedance measurements," Meas. Sci. Technol., vol. 26, no. 4, p. 045601, Mar. 2015.

[22] S. Nukaya, T. Shino, Y. Kurihara, K. Watanabe, and H. Tanaka, "Noninvasive bed sensing of human biosignals via piezoceramic devices sandwiched between the floor and bed," IEEE Sensors J., vol. 12, no. 3 , pp. 431-438, Mar. 2012.

[23] E. S. de Freitas and F. G. Baptista, "Experimental analysis of the feasibility of low-cost piezoelectric diaphragms in impedance-based SHM applications," Sens. Actuators A, Phys., vol. 238, pp. 220-228, Feb. 2016

[24] C. Liang, F. P. Sun, and C. A. Rogers, "Coupled electro-mechanical analysis of adaptive material systems-determination of the actuator power consumption and system energy transfer," J. Intell. Mater. Syst. Struct., vol. 5, no. 1, pp. 12-20, Jan. 1994.

[25] D.-S. Wang, X.-M. Zhou, H.-Y. Song, and H.-P. Zhu, "A twodimensional electromechanical impedance model and experimental verification of a PZT transducer," in Proc. Symp. Piezoelectricity, Acous. Waves, Device Appl. (SPAWDA), Xi'an, China, Oct. 2016, pp. 261-264.

[26] V. G. M. Annamdas and C. K. Soh, "Three-dimensional electromechanical impedance model. I: Formulation of directional sum impedance," J. Aerosp. Eng., vol. 20, no. 1, pp. 53-62, Jan. 2007.

[27] Y. Y. Lim and C. K. Soh, "Towards more accurate numerical modeling of impedance based high frequency harmonic vibration," Smart Mater. Struct., vol. 23, no. 3, p. 035017, Feb. 2014.

[28] M. Deshpande and L. Saggere, "An analytical model and working equations for static deflections of a circular multi-layered diaphragmtype piezoelectric actuator," Sens. Actuators A, Phys., vol. 136, no. 2, pp. 673-689, May 2007.

[29] Y. Hu, X. Liang, and W. Wang, "A theoretical solution of resonant circular diaphragm-type piezoactuators with added mass loads," Sens. Actuators A, Phys., vol. 258, pp. 74-87, May 2017.

[30] L. T. Gomes, "Effect of damping and relaxed clamping on a new vibration theory of piezoelectric diaphragms," Sens. Actuators A, Phys., vol. 169, no. 1, pp. 12-17, Sep. 2011.

[31] R. Z. M. da Silveira, L. M. Campeiro, and F. G. Baptista, "Performance of three transducer mounting methods in impedance-based structural health monitoring applications," J. Intell. Mater. Syst. Struct., to be published, 2017, doi: 10.1177/1045389X17689942.

[32] W. Huang et al., "A Novel ultrasound technique for non-invasive assessment of cell differentiation," IEEE Sensors J., vol. 16, no. 1, pp. 61-68, Jan. 2016.

[33] H. Antlinger, S. Clara, R. Beigelbeck, S. Cerimovic, F. Keplinger, and B. Jakoby, "A differential pressure wave-based sensor setup for the acoustic viscosity of liquids," IEEE Sensors J., vol. 16, no. 21, pp. 7609-7619, Nov. 2016.

[34] F. G. Baptista and J. V. Filho, "Optimal frequency range selection for PZT transducers in impedance-based SHM systems," IEEE Sensors J., vol. 10, no. 8, pp. 1297-1303, Aug. 2010.

[35] T. Kamas and M. Tekkalmaz, "Coupled field modeling of E/M impedance of piezoelectric wafer active sensor for cataphoretic coating thickness measurement," Smart Mater. Struct., vol. 26, no. 4, p. 045035, Mar. 2017.

[36] X. Dong et al., "A new equivalent circuit for piezoelectrics with three losses and external loads," Sens. Actuators A, Phys., vol. 256, pp. 77-83, Apr. 2017
[37] F. G. Baptista, J. V. Filho, and D. J. Inman, "Sizing PZT transducers in impedance-based structural health monitoring," IEEE Sensors J., vol. 11, no. 6 , pp. 1405-1414, Jun. 2011.

[38] F. G. Baptista and J. V. Filho, "Transducer loading effect on the performance of PZT-based SHM systems," IEEE Trans. Ultrason., Ferroelect., Freq. Control, vol. 57, no. 4, pp. 933-941, Apr. 2010.

[39] Murata Manufacturing, Piezoelectric Ceramics ('PIEZOTITE') Sensors, document Catalog No. P19E-9, 2008.

[40] PSI-5A4E Single Layer Disks, accessed on May 19, 2017. [Online] Available: http://www.piezo.com/prodsheet3disk5A.html

[41] F. G. Baptista and J. V. Filho, "A new impedance measurement system for PZT-based structural health monitoring," IEEE Trans. Instrum. Meas., vol. 58, no. 10, pp. 3602-3608, Oct. 2009.

[42] J. Ilg, S. J. Rupitsch, and R. Lerch, "Impedance-based temperature sensing with piezoceramic devices," IEEE Sensors J., vol. 13, no. 6, pp. 2442-2449, Jun. 2013.

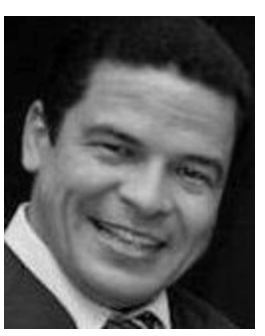

Everaldo S. de Freitas (M'17) was born in Presidente Venceslau, Brazil, in 1978. He received the B.S. degree in computer engineering from the Unilins-University Center of Lins, Lins, Brazil, in 2002. He is currently pursuing the $\mathrm{Ph} . \mathrm{D}$. degree with São Paulo State University (UNESP), Bauru, Brazil, under the supervision of Prof. F. Baptista, with a focus on the field of structural health monitoring based on the electromechanical impedance principle using low-cost piezoelectric transducers. He is a Professor of Automation and Electrical Engineering with the Unilins-University Center of Lins. He is also a Researcher with the Department of Electrical Engineering, UNESP.

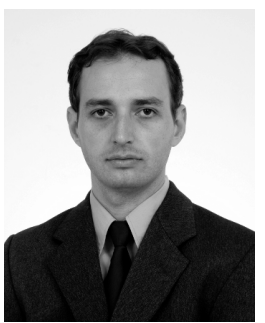

Fabricio G. Baptista (S'08-M'11) received the B.S. and Ph.D. degrees in electrical engineering from São Paulo State University (UNESP), Brazil, in 2006 and 2010, respectively. From 2010 to 2011, he was a Visiting Scholar with the Center for Intelligent Material Systems and Structures, Virginia Tech, Blacksburg, VA, performing research in the field of structural health monitoring and piezoelectric transducers. He is currently an Assistant Professor with the Department of Electrical Engineering, UNESP, Bauru, where he coordinates the Laboratory of Transducers and Data Acquisition, working in the field of piezoelectricity, structural health monitoring, signal processing, data acquisition, and electronic instrumentation.

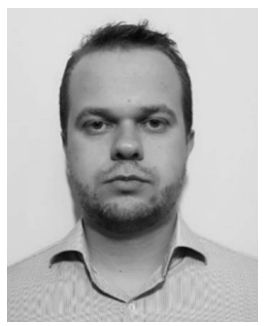

Danilo E. Budoya (S'12-M'17) was born in Bauru, Brazil, in 1989. He received the B.S. degree in electrical engineering from São Paulo State University (UNESP), São Paulo, Brazil, in 2016, where he is currently pursuing the master's degree. He is also a Researcher with the Laboratory of Transducers and Data Acquisition, Department of Electrical Engineering, UNESP, where he works in the area of structural health monitoring, signal processing, data acquisition, and electronic instrumentation.

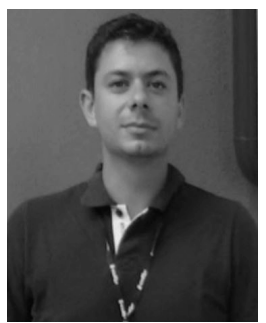

Bruno A. de Castro (S'08-M'16) received the B.S. and M.Sc. degrees in electrical engineering from São Paulo State University, Bauru, Brazil, in 2012 and 2016, respectively, where he is currently pursuing the $\mathrm{Ph} . \mathrm{D}$. degree in electrical engineering. His research field focus is on structural health monitoring, failure diagnostics of civil, mechanical structures, intelligent systems, data acquisition, electronic instrumentation, acoustic emission, piezoelectricity, instrumentation, and power quality. 